\title{
A Semi-Deterministic Channel Model for VANETs Simulations
}

\author{
Jonathan Ledy, ${ }^{1}$ Hervé Boeglen, ${ }^{2}$ Anne-Marie Poussard, ${ }^{1}$ \\ Benoît Hilt, ${ }^{2}$ and Rodolphe Vauzelle ${ }^{1}$ \\ ${ }^{1}$ Laboratoire XLIM-SIC, UMR CNRS 6172, Université de Poitiers, 86034 Poitiers, France
}

${ }^{2}$ Laboratoire MIPS-GRTC, Université de Haute Alsace, 68000 Colmar, France

Correspondence should be addressed to Jonathan Ledy, ledy@sic.univ-poitiers.fr

Received 15 October 2010; Revised 24 May 2011; Accepted 2 June 2011

Academic Editor: Athanasios Panagopoulos

Copyright (c) 2012 Jonathan Ledy et al. This is an open access article distributed under the Creative Commons Attribution License, which permits unrestricted use, distribution, and reproduction in any medium, provided the original work is properly cited.

\begin{abstract}
Today's advanced simulators facilitate thorough studies on Vehicular Ad hoc NETworks (VANETs). However the choice of the physical layer model in such simulators is a crucial issue that impacts the results. A solution to this challenge might be found with a hybrid model. In this paper, we propose a semi-deterministic channel propagation model for VANETs called UM-CRT. It is based on CRT (Communication Ray Tracer) and SCME_-UM (Spatial Channel Model Extended_-Urban Micro) which are, respectively, a deterministic channel simulator and a statistical channel model. It uses a process which adjusts the statistical model using relevant parameters obtained from the deterministic simulator. To evaluate realistic VANET transmissions, we have integrated our hybrid model in fully compliant $802.11 \mathrm{p}$ and $802.11 \mathrm{n}$ physical layers. This framework is then used with the NS-2 network simulator. Our simulation results show that UM-CRT is adapted for VANETs simulations in urban areas as it gives a good approximation of realistic channel propagation mechanisms while improving significantly simulation time.
\end{abstract}

\section{Introduction}

Vehicular Ad hoc NETworks (VANETs) are a very promising research area interesting the scientific community, car manufacturers, and mobile telephony operators. Vehicular applications should be thoroughly tested before they are deployed in the real world. Because the setup of experimental VANETs would imply huge investments, computer simulations are generally preferred.

One of the major issues when using simulators for VANETs concerns the vehicular environment and therefore the realistic modeling of the wireless propagation channel. Indeed, there are still several problems linked to the impact of the mobility and the traffic density on channel statistics yet to solve, for example, packets loss, rate of flow, frequency correlation, and amplitude distribution.

Many research and development works relating to routing [1], communication robustness [2], and information dissemination in VANETs [3] show results obtained with simulations involving very basic radio propagation models available in simulation tools (Friis and two-ray ground models, e.g.). The consequence of the mobility on the physical layer is most of the time treated in a simplistic and consequently not quite realistic manner. This can lead to erroneous results [4]. Moreover, one finds very few effective and robust channel models which take into account the mobility and especially the transmission environment.

From this, one can understand that the radio propagation model used by the network simulation tool is a key factor in MANETs (Mobile Ad Hoc NETworks) and particularly in the VANETs subclass. Developing a radio channel model, which would describe the realistic radio channel conditions as accurately as possible, has been a continuous challenge. This is precisely what this work addresses.

There already exist reliable channel models which are customizable according to the environment [5], but most of them are dedicated to mobile telephony. In parallel, one finds research works presenting deterministic channel models $[6,7]$ which are based on ray-tracing or ray-launching methods which allow a realistic modeling of the channel. Unfortunately, these models require very high processing times.

As far as VANETs are concerned, deterministic channel models are not suitable because of the high mobility, the 
diversity of the environment encountered, and the high number of communicating nodes. The study of the higher layers of the OSI model (in particular the Network and Application layers) requires a low simulation time (i.e., a couple of minutes) in order to allow statistical analyses on large simulation series. To answer the challenge of channel modeling in VANETs, several works propose various methods which can be classified in two categories according to the research domain of their authors.

In the network community, Dhoutaut et al. [8] propose a propagation model based on Markov chains elements and real world experiments which is able to generate packet losses in a very realistic way. Later on, Han and AbuGhazaleh proposed another method based on Finite State Markov model [9]. These models are half-way between very detailed models using ray-tracing with computationally intensive algorithms and models using theoretical analysis where physical phenomena are only handled in an aggregate manner. But according to the authors, their models are not yet able to make a clear relation with a real environment.

In the physical channel modeling community, one can find different statistical channel models which have been derived from intensive measurement campaigns. The stochastic parameters of these models are extracted from the measurement data. It has been shown that the measured amplitude samples follow Rice, Rayleigh, or Weibull distributions [10]. In the case of vehicular channels there exist such type of channel models which have been designed by Acosta-Marum and Ingram for the validation of the 802.11p standard $[11,12]$. It is a classical tapped delay line Wide Sense Stationary Uncorrelated Scattering (WSSUS) channel model. In [10], Sen and Matolak propose channel models which take into account the sudden appearance/disappearance of scatterers (moving obstacles) by modeling them as firstorder, two-state Markov chains. In [13] Keredal et al. propose a geometry-based stochastic MIMO model for Vehicleto-Vehicle (V2V) communications. However, as stated by Molisch in [14], there is only a small amount of V2V channel measurements available which "does not allow the derivation of statistically significant statements about real-world V2V channels". In conclusion, extreme care has to be taken when choosing and parameterizing available statistical channel models for VANETs.

Another fundamental topic in VANETs simulation concerns the mobility model used for simulations. Many works, like the working by Marfia et al. [15], show the importance of realistic mobility models. Indeed, the use of nonspecific mobility models employed in VANETs simulations may provide bad results, because they ignore the typical behavior of the nodes in this kind of network. In conclusion, a combination of realistic radio wave propagation models and realistic mobility models is a large step towards more realistic simulation environments as shown by Günes et al. [16]. In our work, the selected simulation tools are the VANET-specific mobility generator VanetMobiSim [17] and the generic network simulation tool NS-2 [18].

The rest of this paper is organized as follows. In Section 2, we present the components of UM-CRT that are, respectively, the statistical SCME-UM model and the realistic
CRT simulator. In Section 3, we give a detailed presentation of our semi-deterministic model, UM-CRT. Our framework including implementation and the simulation of the 802.11 standard is also presented in this section. Section 4 is dedicated to the evaluation of our model. Finally, Section 5 concludes this paper and deals with future works.

\section{Towards a Semi-Deterministic Model}

Statistical and deterministic channel models are the two common ways to describe the radio channel behavior in VANETs simulations. In this section, we describe an example of each of these approaches: for the statistical one, the Spatial Channel Model Extended in its Urban Microenvironment (SCME-UM) is described and, for the deterministic one, the Communication Ray Tracer (CRT) simulator is presented. We then analyze their main characteristics in order to propose a new semi-deterministic solution which benefits from the advantages of these two classical approaches. This proposition is called UM-CRT because it is based on respectively, SCME-UM and CRT.

2.1. The Spatial Channel Model Extended (SCME). The Spatial Channel Model Extended (SCME) statistical channel model is an evolution of the 3GPP Spatial Channel Model (SCM) [19]. It has been developed within the European WINNER project [20] for the simulation of B3G systems. The SCM model is limited to the simulation of systems at $2 \mathrm{GHz}$ for a maximum transmission bandwidth of $5 \mathrm{MHz}$, whereas its extension, SCME for SCM Extended, allows for the simulation of systems at 2 and $5 \mathrm{GHz}$ for a maximum transmission bandwidth of $100 \mathrm{MHz}$ [21].

SCM and SCME are so-called geometric models for which scatterers are placed stochastically in the simulation scene. SCME considers clusters of scatterers. Each cluster corresponds to a resolvable path. Each path is made up of several nonresolvable subpaths. Figure 1 is a typical example showing the main geometrical parameters used by the model where a Base Station (BS) antenna array communicates with a Mobile Station (MS) antenna array. This example shows only one cluster labeled n. A subpath is also shown (labeled $\mathrm{m})$.

SCME is a natively Multiple Input Multiple Output (MIMO) model. It allows for the simulation of three types of environments: Urban Macrocell, Suburban Microcell (distance between MS and BS $3 \mathrm{~km}$ maximum), and Urban Microcell (distance between MS and BS of $1 \mathrm{~km}$ maximum). In the context of urban VANETs, because of intervehicular distances less than one kilometer we have chosen the Urban Microcell (UM) environment. The authors of [21] provide a Matlab implementation of the SCME model [22] which we have used in our framework. This piece of software generates Channel Impulse Responses (CIR) which can then be used in a digital communication chain.

2.2. The Communication Ray Tracer (CRT) Simulator. Let us now describe the Communication Ray Tracer (CRT) software. It is a deterministic propagation simulator developed by the Xlim-SIC laboratory from the University of 


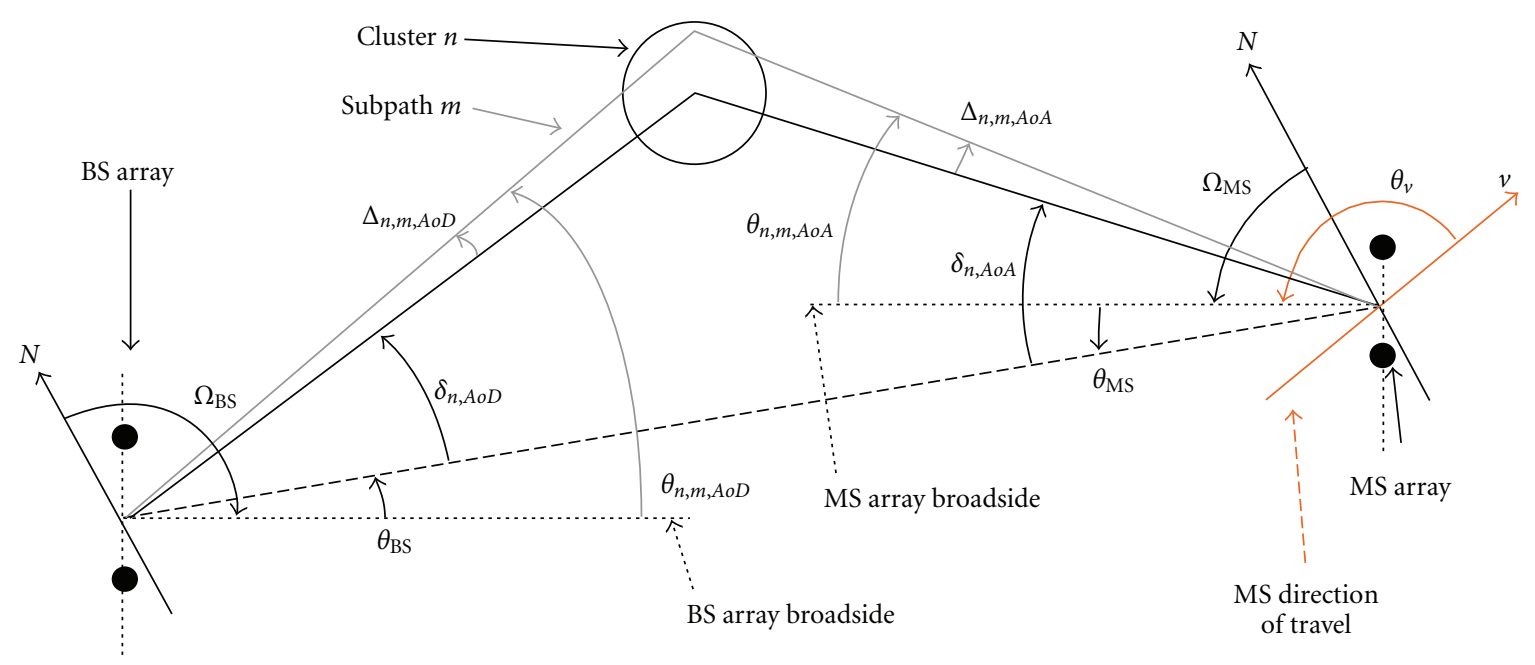

FIGURE 1: Geometric parameters of the SCME model.

Poitiers (France) [23]. CRT is based on an optimized 3D deterministic ray-tracing method to model the propagation of radio waves in real environments (outdoor and indoor). Thus, CRT takes into account all the characteristics (geometric and electric) of the environment and provides the information about the multipath phenomenon, where each path is characterized by its attenuation, delay, angular directions, phase shift, and polarization. In this way, we obtain a complete characterization of the narrow-band and wide-band channel. This provides a realistic approach of the multi-path propagation mechanisms. CRT has been validated by several measurement campaigns [23] and will therefore be considered as our reference.

Figure 2 shows an example of a multi-path propagation simulation in a 3D urban environment using CRT. Each white line is one path followed by the radio waves between the transmitter and the receiver defined by a black circle in Figure 2. The CRT simulation procedure is as follows. Firstly, the user chooses an environment and places the communicating nodes into that scene. In a second stage, the nodes are associated with a moving trajectory. Then, CRT calculates the CIRs for all the communicating nodes in the simulation scenario.

In order to reduce the computation time in a context of mobility, an optimization based on the stationarity property of the channel [24] has been studied. It has been shown that a stationarity area of 8 meters can be considered.

2.3. Basis of the Proposed Semi-Deterministic Model for VANETs. The presentation of the two previous models shows several key elements.

The SCME-UM statistical model is very efficient to produce CIRs in environments modeled by several clusters placed randomly in the scene. However, it does not take into account the geometrical specificities of real environments. On the contrary, CRT is able to provide CIRs directly connected with the environment by a complete modeling of the interactions between radio waves and building. However,

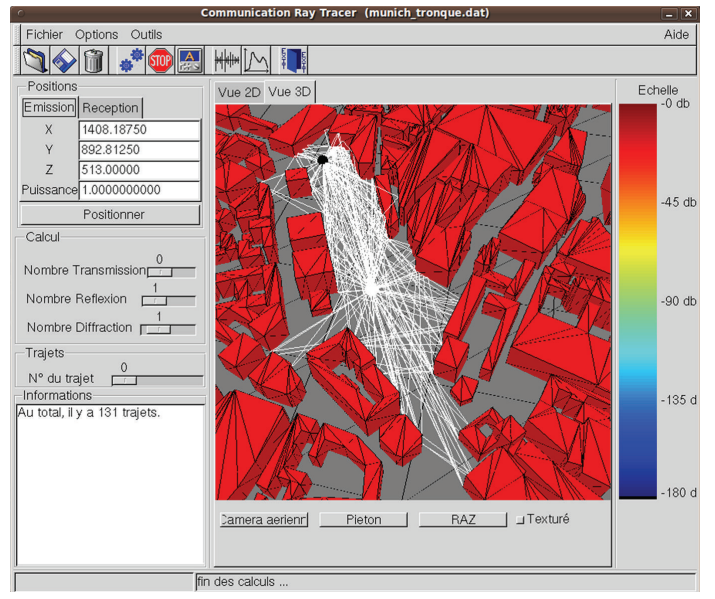

FIGURE 2: A CRT simulation of multipath propagation in a realistic 3D urban environment.

it necessitates an important computation time for each CIR calculation. Therefore, for VANETs applications, which introduce the mobility for each node and a significant number of possible radio links between the nodes in the network, a deterministic solution leads to a huge computation time.

To address this problem, we propose in this paper a hybridisation of the two models presented previously named UM-CRT. Indeed, we will combine at the same time the major wave propagation phenomena (existence or not of the direct path) with its deterministic component and the low computation time allowed by its statistical one.

\section{The Semi-Deterministic Channel Model}

3.1. The UM-CRT Semi-Deterministic Channel Model. According to the principle presented in the previous section, UM-CRT is created from the association of two models. Figure 3 shows the relationship between UM-CRT and both CRT and SCME-UM, respectively, in the left and right parts 


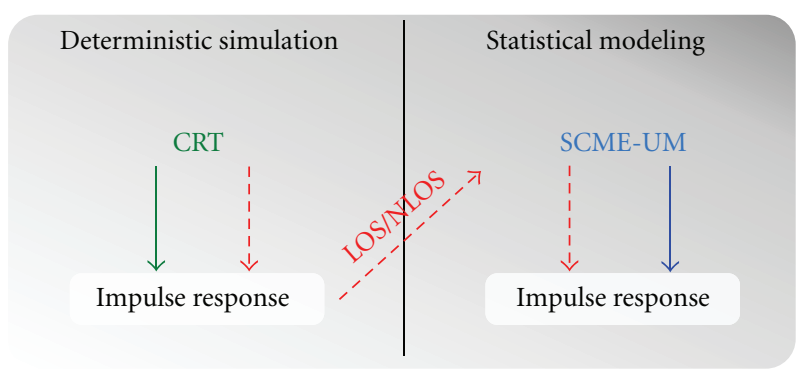

Figure 3: Principle of the UM-CRT model.

with fine arrows. UM-CRT is depicted with bold dashed arrows.

Classically, for all radio links existing between nodes, on the one hand, CRT computes CIRs according to the simulation of all the received multi-paths according to an environment modeled in 3D. On the other hand, SCME-UM provides statistically generated CIRs.

Here, we propose to limit the search path by ray-tracing only to the direct path because it is well known that this path has the main impact on the received signal. This limitation has two advantages.

(i) It takes into account the geometrical characteristic of the considered environment: we can determine the Line of Sight (LOS) and Nonline of Sight (NLOS) radio links.

(ii) It considerably reduces the computation time of the deterministic simulation.

On this basis, it is possible to generate representative CIRs with SCME-UM.

Notice that complete multi-path simulations with CRT are possible in order to exploit other information included in the deterministic CIRs such as delay spread. But it will be very time consuming.

Moreover, in order to reduce again the computation time, we propose to consider the stationarity property of the channel introduced previously. We assume that a CIR of a radio link remains constant when the move of its extremities is less than 8 meters in relation to a reference position. So we do not compute the CIRs at each time but only for a finite number of distances between the transmitter and the receiver. In practice, as the SCME-UM is limited to 600 meters between the transmitter and the receiver, we consider a set of 90 distances to calculate the CIRs. These CIRs can be precalculated in order to accelerate the computation time for statistical studies of VANETS performance.

To summarize, for a VANETs scenario based on a set of vehicles, UM-CRT allows computing a statistical CIR of each radio link between two mobile nodes, at each time, according to a LOS/NLOS deterministic analysis.

3.2. The UM-CRT Framework. In order to evaluate the performance of a VANETs scenario in a realistic environment, it is necessary to introduce real transmission conditions in a network simulator. We consider here the NS2 platform.
These transmission conditions are based on the channel model and on the specific digital communication chain considered. This constitutes a realistic physical layer.

Firstly, we explain the considered physical layer. Secondly, we introduce the UM-CRT framework in an NS2 context. With this framework, it is possible to compute the performance analysis according to some QoS metrics (Packet Delivery Ratio, Delay, etc.) in several configurations, as it will be shown in Section 4. Finally, we show the validity (ou accuracy) of Bit Error Rate (BER) calculated with our framework in the Munich city center as an exemple.

Concerning the physical layer in VANETs context, there exists the $802.11 \mathrm{p}$ standard adopted at the the end of 2010 [25]. It is an adaptation of the 802.11a standard for Dedicated Short Range Communication (DSRC) between vehicles. Although 802.11p improves the robustness against channel frequency selectivity, doubling the OFDM symbol time degrades the robustness of the system against time selectivity which depends on the Doppler frequency shift directly related to the vehicles speed.

The $802.11 \mathrm{p}$ standard does not account for the MultipleInput Multiple-Output technology although it is known to improve significantly the reliability and the throughput of data transmission [26-28]. We believe that this issue is going to be a natural evolution of the standard. From the time being, and in order to assess the communication robustness performance of MIMO systems in VANET scenarios, we have decided to use the $802.11 \mathrm{n}$ standard in our simulations where we set up a $2 \times 2$ antenna system. Last but not least, the $802.11 \mathrm{n}$ standard allows transmissions in the $5 \mathrm{GHz}$ frequency band. The $802.11 \mathrm{p}$ and $802.11 \mathrm{n}$ implementations have been written in C++ using the IT++ library [29].

In [30] Hamidouche et al. study the impact of a realistic physical layer on the H.264/AVC video transmission over ad hoc networks in an urban environment. They also propose an error model which is based on a Bit Error Rate (BER) computation. We will make use of this error model into our model-building process.

From the impulse response calculated by SCME-UM, we use the 802.11 physical layer described previously to calculate a BER. Each BER value gives an accurate information about the quality of the communication between two nodes.

In order to evaluate the accuracy of our realistic BER computation approach, we consider the $802.11 \mathrm{p}$ standard in the environment in which we will run our simulations, that is to say a VANETs scenario with 40 vehicles moving in the Munich city center. Figure 4 shows the BER evolutions according to SNR observed for all radio links associated to the scenario. The red curve is obtained with the UMCRT model and the blue one with the CRT simulation. The last one is considered as the reference because the channel impulse responses are computed deterministically.

We can observe that the results are very close and consequently the approach proposed in our framework is valid in terms of BER.

Finally, this realistic physical layer based on UM-CRT and called UM-CRT framework is introduced in the NS2 


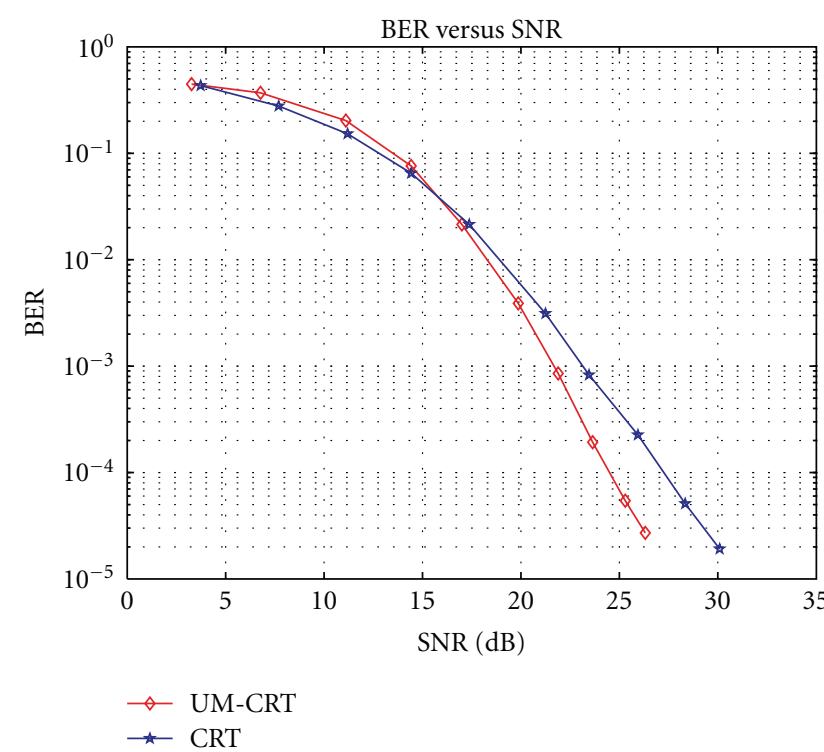

FIGURE 4: BER evolutions according to SNR obtained by UM-CRT and CRT.

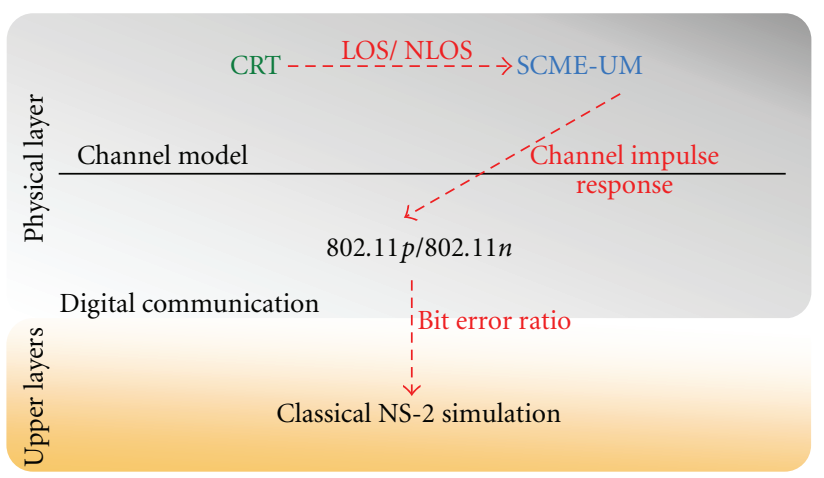

FIGURE 5: The UM-CRT framework.

platform. It communicates with the upper layers. All these steps are summarized in Figure 5.

Figure 6 shows an example of an application of the UM-CRT framework in our simulation environment with buildings shown in red. This figure shows an instantaneous representation of a 40 -vehicle scenario with several communications. The colored lines represent the BER values between each transmitter-receiver couple of vehicles, characterizing the radio link quality. The darker the color, the higher the BER. This tool is useful to determine easily which route between nodes is bad or good.

To conclude, the NS2 platform modified with the UMCRT framework constitutes a VANETs simulator which allows to evaluate QoS performance with realistic transmission conditions in a specific environment.

\section{UM-CRT Evaluation}

To evaluate the UM-CRT propagation model, we compare it with the CRT simulator using NS-2 simulations in a typical urban environment: that is, the center of Munich City

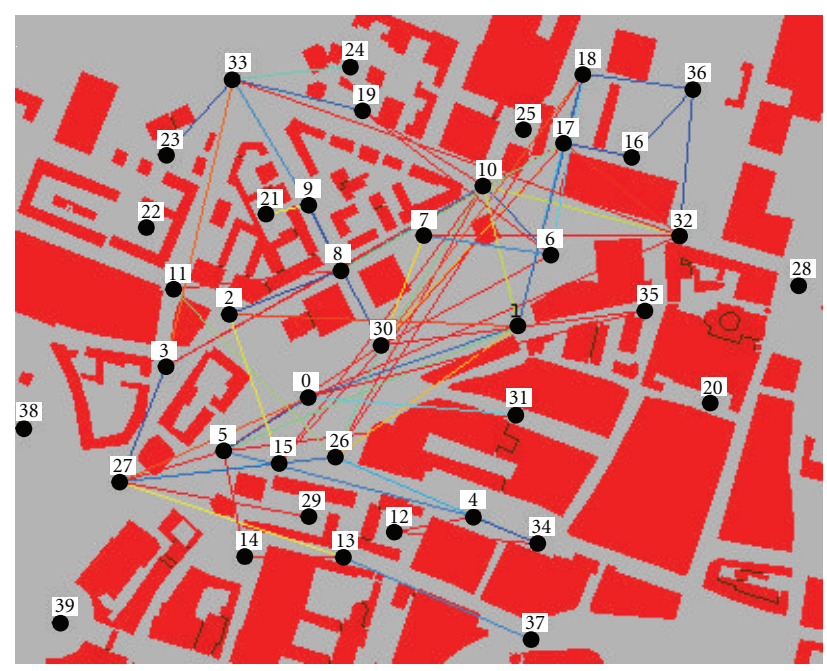

Figure 6: Instantaneous representation of the radio link quality in terms of BER between vehicles in the Munich city center.

(cf. Figure 6). As mentioned above, CRT will be our reference model and can be considered realistic enough to match real world implementation results.

4.1. Accuracy Evaluation. Please note that in this evaluation, the results of the statistical SCME-UM model used alone are not presented because they do not take into account a real propagation environment. LOS and NLOS results will always either be nearly perfect ( $\sim 100 \%$ of packets reach their destination) or bad ( $\sim 0 \%$ of packets reach their destination).

The simulations were run in SISO and MIMO modes in the $5 \mathrm{GHz}$ band. In order to have enough different cases for comparing UM-CRT to CRT, we ran simulations with different VANET realistic mobility scenarios generated by VanetMobiSim. Each of them is defined with different starting points, traffic lights configurations, and mobilities. The mobility is random and the nodes' speed is variable with time and limited by 3 maximum allowed speeds $(0,8$, and $15 \mathrm{~m} / \mathrm{s})$. The routing protocol we used is AODV in its basic setup. The traffic generated for inter-node communications is UDP based. The simulations were performed on a Linux Mandriva system with an updated NS-2.29 simulator version.

We first compare UM-CRT to CRT in the SISO mode (802.11p). The comparison is done in terms of packet delivery ratio (PDR) between a transmitter and a receiver. This is a common criterion used in VANETs performance evaluation. We also compare them in terms of number of hops and end-to-end delay. We finally evaluate how MIMO impacts the PDR (802.11n case). Note that in these evaluations we present averaged results for every simulation over 5 different 40 -second simulation time.

Figure 7 shows that the PDR varies in an important manner depending to the maximum allowed speed. Remember that our main goal is to compare the proximity of the results of UM-CRT and CRT in every situation. We can observe that the higher the speed is, the closer the PDR results are. We can also see that in the static situation $(0 \mathrm{~m} / \mathrm{s})$, UM-CRT results 


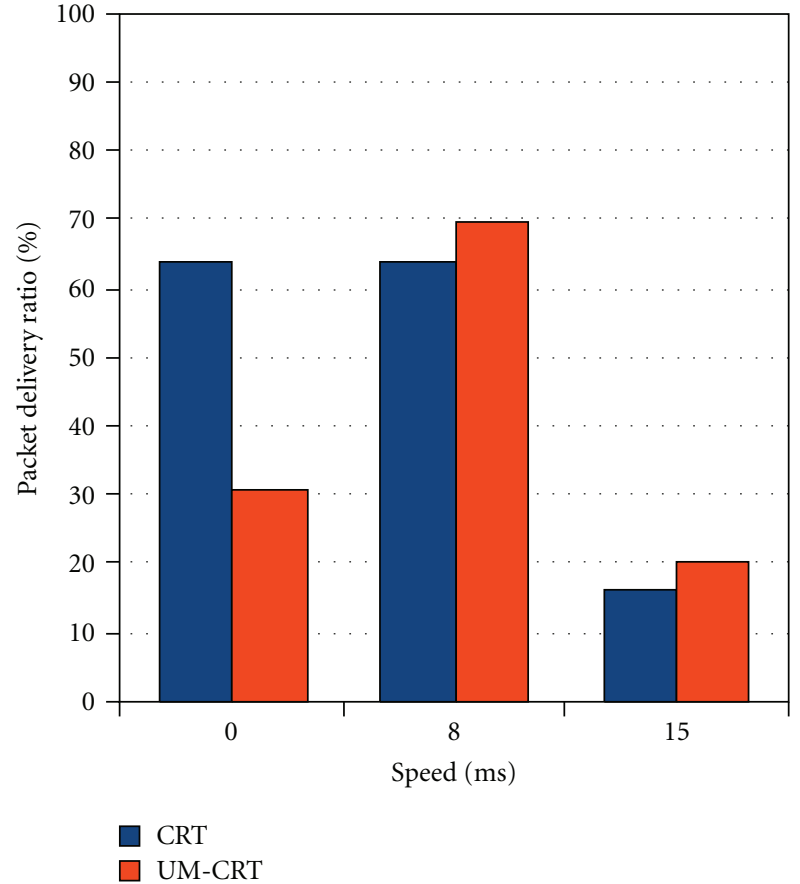

FIGURE 7: UM-CRT PDR evaluation.

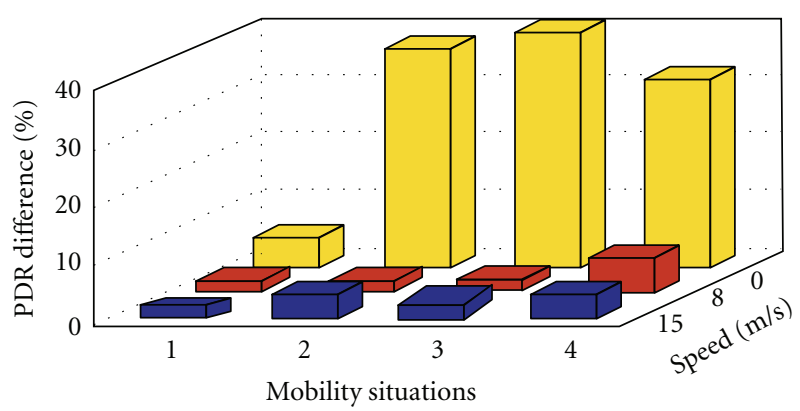

Figure 8: UM-CRT versus CRT PDR in four mobility situations.

do not match the CRT reference results. Additionally, the known negative influence of the speed on the PDR can be observed in the $15 \mathrm{~m} / \mathrm{s}$ part of the figure.

In Figure 8 we have a global view of the PDR difference between UM-CRT and CRT in several mobility situations. This confirms the similarity between UM-CRT and CRT and therefore the realism of our semi-deterministic model when we are in nonstatic situations. In the cases with mobility, the difference between the two models is less than $10 \%$.

Figures 9 and 10 confirm that concerning the end-toend delay and the average number of hops UM-CRT matches better CRT; that is, it becomes more realistic, when the speed is higher. If we look further, the similarity between the two models can also be observed through an increasing delay and average number of hops that both increase when the speed increases. For these two parameters, the difference between the two models decreases above 0,1 seconds for the delay and can be considered as very similar for the number of hops, when we are not in a static situation.

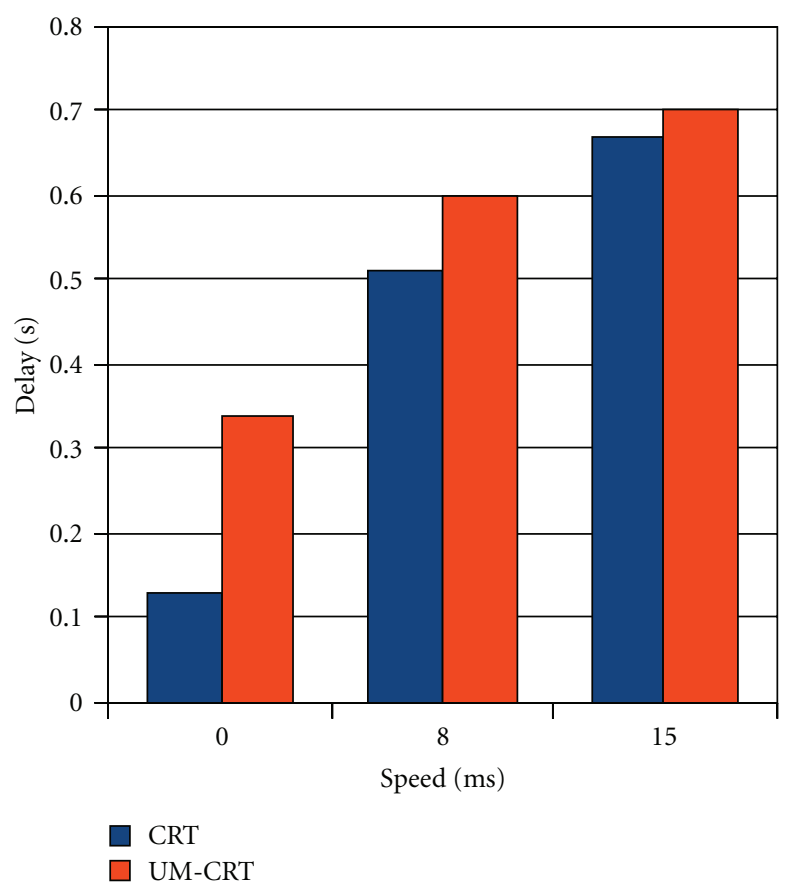

FIgURE 9: Delay evaluation.

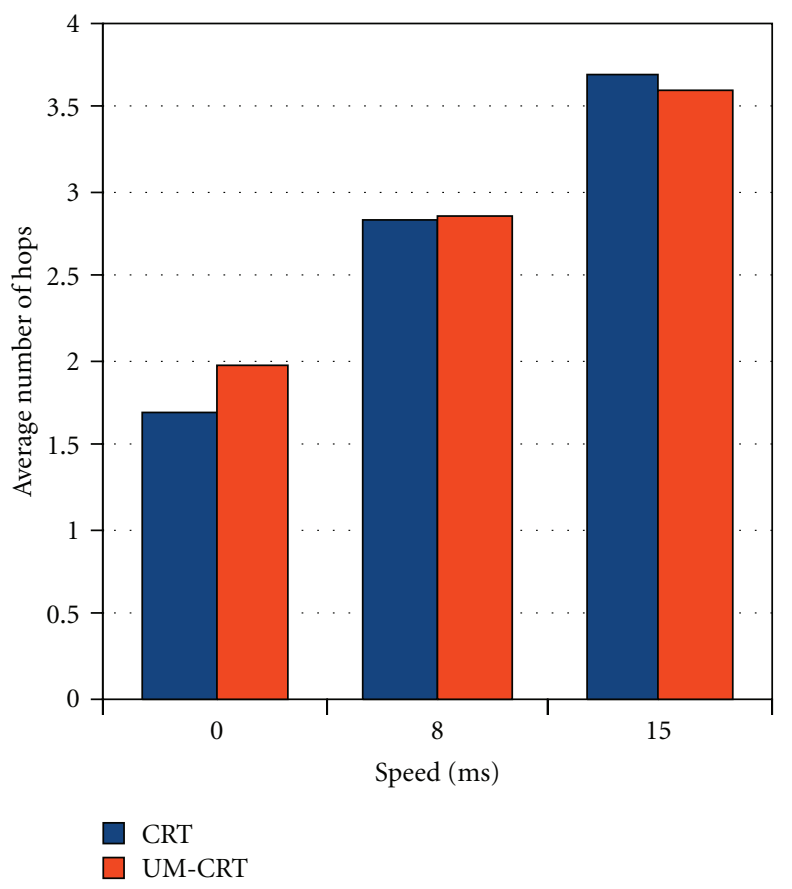

FIgURE 10: Average number of hops evaluation.

Results have the same trend in the case of a static situation in the simulations. This is a current limitation of our model which is not suitable for null speed. In this case, the LOSNLOS criterion is not suitable alone to produce results that match the deterministic model.

To summarize, when we are not in a static situation, the UM-CRT model gives results quite similar to CRT in all 
TABLe 1: Simulation time comparison.

\begin{tabular}{|c|c|c|c|c|c|c|c|c|c|c|c|c|}
\hline \multirow[b]{2}{*}{ Scenario } & \multicolumn{4}{|c|}{ CRT } & \multicolumn{4}{|c|}{ UM-CRT } & \multicolumn{4}{|c|}{ SCME-UM } \\
\hline & 1 & 2 & 3 & 4 & 1 & 2 & 3 & 4 & 1 & 2 & 3 & 4 \\
\hline Preprocessing Full CRT (h) & 18 & 18 & 17 & 18 & & & & & & & & \\
\hline Preprocessing LOS/NLOS (h) & & & & & 1 & 1 & 1 & 1 & 1 & 1 & 1 & 1 \\
\hline Simulation $(\mathrm{h})$ & 57 & 65 & 55 & 63 & 0.5 & 0.5 & 0.5 & 0.5 & 0.5 & 0.5 & 0.5 & 0.5 \\
\hline TOTAL Time $(\mathrm{h})$ & 75 & 83 & 72 & 81 & 1.5 & 1.5 & 1.5 & 1.5 & 1.5 & 1.5 & 1.5 & 1.5 \\
\hline
\end{tabular}

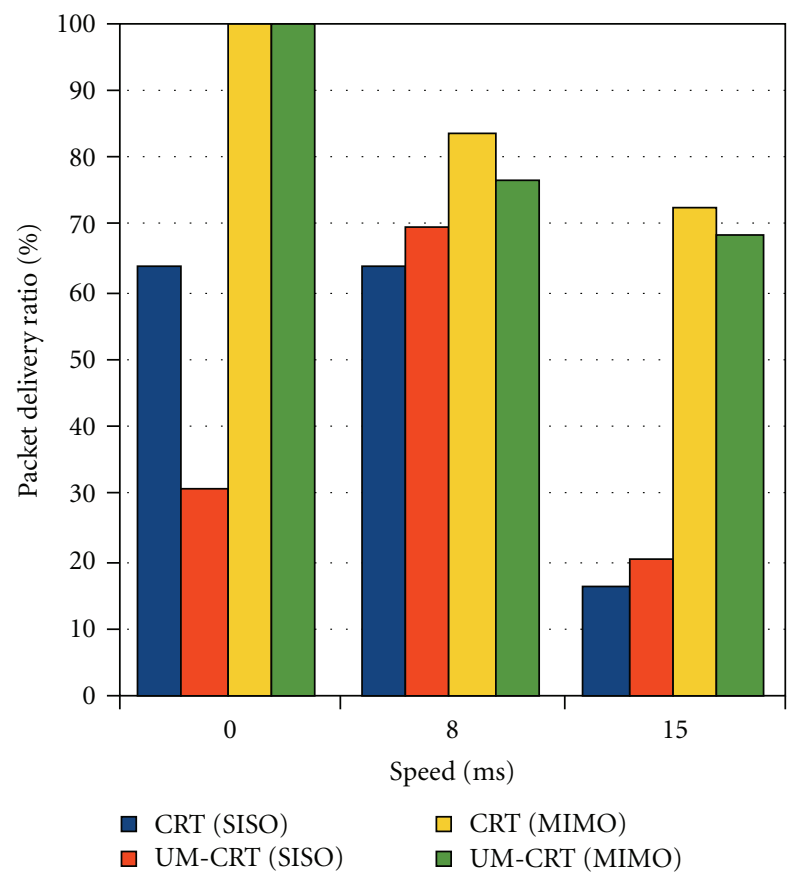

FIgURE 11: MIMO Impact.

situations. This can be explained by the number of statistical outcomes that increase because of the mobility. So we can conclude that the UM-CRT model gives results quite close to the deterministic model.

Furthermore, as the speed gets higher, one can see that the channel deteriorates (the received packets rate decreases) and that it is more difficult to achieve a reliable communication (delay and average number of hops increase). We can see that, for a maximum speed of $15 \mathrm{~m} / \mathrm{s}$, the received packet rate does not exceed $50 \%$. This is a second expression of the determinism that our statistically based model produces.

We will now see the impact of the use of UM-CRT in a MIMO mode instead of a SISO mode.

The impact of the MIMO mode on the packet delivery ratio is presented in Figure 11. It confirms the tendencies observed in the SISO case. The UM-CRT model gives quite similar results to CRT for the MIMO mode too. However, there is an exception in the static case for MIMO: CRT and UM-CRT results match perfectly at 0 millisecond whereas they do not at 8 milliseconds or 15 milliseconds. This can be explained by the robustness of the channel which has improved compared to the SISO case. It is therefore possible to obtain $100 \%$ of received packets even with fixed nodes.
Indeed, results between CRT and UM-CRT are close. As expected, we can also notice that a MIMO channel is more robust than a SISO one. The received packets rates are better in MIMO cases, no matter the nodes speed. Approximately $70 \%$ of the packets are received with a $15 \mathrm{~m} / \mathrm{s}$ speed in this scenario for the MIMO mode, whereas it is less than $50 \%$ for the SISO mode.

From these results, one can conclude that the MIMO mode improves the transmission's robustness in VANETs. So MIMO technology allows reducing transmission power with a packet delivery ratio equal to the SISO mode in order to limit perturbations, or it can help to improve the packet delivery ratio. In both cases, MIMO technology seems to be very interesting to answer VANETs' challenges.

4.2. Computation Time Evaluation. As shown in Section 4.1, our semi-deterministic propagation model is not only very realistic (except for static nodes situations) but it also decreases the simulation time.

In Table 1, one can observe that each CRT simulation lasts at least three days (equivalent to 40-second simulated time in NS-2) whereas in the case of SCME-UM this reduces to only 1.5 hours. Section 4.1 has shown the realism of UMCRT. Table 1 showed that the computation time for UMCRT has been reduced significantly. This can be explained by the principle used in the model (see Section 3) which consists in reading a cache containing CRT and SCME-UM impulse responses which are then used for BER computations.

\section{Conclusion and Future Works}

In this paper we have presented UM-CRT, a semideterministic channel propagation model for VANETs. UMCRT, which was integrated into the NS-2 network simulator, is based on the stochastic SCME-UM model and the deterministic CRT channel simulator.

The implementation of this new model allows to run network simulations in a very fast way. Indeed the computation time is reduced from more than 70 hours to less than 2 hours. This makes UM-CRT quite suitable for VANETs simulations having a large number of highmobility nodes. Moreover, our results showed UM-CRT to be appropriate for mobility scenarios and realistic vehicular networks simulations, typically urban scenarios.

Furthermore, results show that UM-CRT is also adapted for the MIMO technology. As expected, simulations involving this configuration have clearly showed the robustness of the MIMO scheme compared to SISO one. 
We currently focus our work on the selection of new relevant criteria extracted from the CIR such as the RMS delay spread or the link capacity.

\section{References}

[1] M. Boban, G. Misek, and O. K. Tonguz, "What is the best achievable QoS for unicast routing in VANET?" in Proceedings of the IEEE Globecom Workshops, pp. 1-10, New Oreleans, La, USA, December 2008.

[2] S. Y. Wang, "The effects of wireless transmission range on path lifetime in vehicle-formed mobile ad hoc networks on highways," in Proceedings of the IEEE International Conference on Communications, vol. 5, pp. 3177-3181, Seoul, Korea, May 2005.

[3] S. Yousefi, S. Bastani, and M. Fathy, "On the performance of safety message dissemination in vehicular ad hoc networks," in Proceedings of the 4th European Conference on Universal Multiservice Networks, pp. 377-387, Toulouse, France, February 2007.

[4] F. J. Martinez, C. K. Toh, J. C. Cano, C. T. Calafate, and P. Manzoni, "Realistic radio propagation models (RPMs) for VANET simulations," in Proceedings of the IEEE Wireless Communications and Networking Conference, Budapest, Hungary, April 2009.

[5] 3GPP, "Spatial channel model for MIMO simulations," TR 25.996 V9.0.0 (2009-12), http://www.3gpp.org/ftp/Specs/ archive/25_series/25.996/25996-900.zip.

[6] I. Stepanov and K. Rothermel, "On the impact of a more realistic physical layer on MANET simulations results," Elsevier Ad Hoc Networks Journal, vol. 6, no. 1, pp. 61-78, 2006.

[7] R. Delahaye, A.-M. Poussard, Y. Pousset, and R. Vauzelle, "Propagation models and physical layer quality criteria influence on ad hoc networks routing," in Proceedings of the 7th International Conference on Intelligent Transport Systems Telecommunications, pp. 433-437, Sophia Antipolis, France, June 2007.

[8] D. Dhoutaut, A. Régis, and F. Spies, "Impact of radio propagation models in vehicular ad hoc networks simulations," in Proceedings of the 3rd ACM International Workshop on Vehicular Ad Hoc Networks (VANET '06), pp. 40-49, Los Angeles, Calif, USA, September 2006.

[9] S. Han and N. B. Abu-Ghazaleh, "Estimated measurementbased Markov models: Towards flexible and accurate modeling of wireless channels," in Proceedings of the 5th IEEE International Conference on Wireless and Mobile Computing Networking and Communication, pp. 331-337, Marrakech, Morocco, October 2009.

[10] I. Sen and D. W. Matolak, "Vehicle-vehicle channel models for the 5-GHz band," IEEE Transactions on Intelligent Transportation Systems, vol. 9, no. 2, Article ID 4517519, pp. 235-245, 2008.

[11] G. Acosta-Marum and M. A. Ingram, "A BER-based partitioned model for a $2.4 \mathrm{GHz}$ vehicle-to-vehicle expressway channel," Wireless Personal Communications, vol. 37, no. 3-4, pp. 421-443, 2006.

[12] G. Acosta-Marum and M. A. Ingram, "Six time- and frequency- selective empirical channel models for vehicular wireless LANs," IEEE Vehicular Technology Magazine, vol. 2, no. 4, Article ID 4498409, pp. 4-11, 2007.

[13] J. Karedal, F. Tufvesson, N. Czink et al., "A geometry-based stochastic MIMO model for vehicle-to-vehicle communications," IEEE Transactions on Wireless Communications, vol. 8, no. 7, pp. 3646-3657, 2009.
[14] A. F. Molisch, F. Tufvesson, J. Karedal, and C. F. Mecklenbrauker, "A survey on vehicle-to-vehicle propagation channels," IEEE Wireless Communications, vol. 16, no. 6, pp. 12-22, 2009.

[15] G. Marfia, G. Pau, E. De Sena, E. Giordano, and M. Gerla, "Evaluating vehicle network strategies for downtown Portland: opportunistic infrastructure and the importance of realistic mobility models," in Proceedings of the 5th International Conference on Mobile Systems, Applications and Services, pp. 47-51, San Juan, Puerto Rico, USA, June 2007.

[16] M. Günes, M. Wenig, and A. Zimmermann, "Realistic mobility and propagation framework for MANET simulations," in Proceedings of the 6th International Conference on Networking, Atlanta, Ga, USA, 2007.

[17] J. Haerri, F. Filali, C. Bonnet, and M. Fiore, "VanetMobiSim: generating realistic mobility patterns for VANETs," in Proceedings of the 3rd ACM International Workshop on Vehicular Ad Hoc Networks (VANET '06), Los Angeles, Calif, USA, September 2006.

[18] http://www.isi.edu/nsnam/ns.

[19] http://www.3gpp.org/ftp/Specs/html-info/25996.htm.

[20] http://www.ist-winner.org.

[21] D. S. Baum, J. Hansen, G. Del Galdo, M. Milojevic, J. Salo, and P. Kyösti, "An interim channel model for beyond-3G systems: extending the 3GPP spatial channel model (SCM)," in Proceedings of the 61st Vehicular Technology Conference (VTC' 05), vol. 5, pp. 3132-3136, Stockholm, Sweden, June 2005.

[22] http://radio.tkk.fi/en/research/rf_applications_in_mobile_ communication/radio_channel/scme-2006-08-30.zip.

[23] F. Escarieu, V. Degardin, L. Aveneau et al., "3D modelling of the propagation in an indoor environment : a theoretical and experimental approach," in Proceedings of the European Conference on Wireless Technologies (ECWT'01), London, UK, September 2001.

[24] R. Delahaye, Simulation réaliste et efficace de la couche physique pour l'aide au routage des réseaux ad hoc, Ph.D. thesis, University of Poitiers, France, 2007.

[25] IEEE Standard for information technology-Telecommunications and information exchange between systems-Local and metropolitan area networks-Specific requirements, Part 11: Wireless LAN Medium Acces Control (MAC) and Physical layer (PHY) specifications, Amendment 6: Wireless Access in Vehicular Environments, IEEE std 802.11p, 2010.

[26] G. J. Foschini, "Layered space-time architecture for wireless communication in a fading environment when using multielement antennas," Bell Labs Technical Journal, vol. 1, no. 2, pp. 41-59, 1996.

[27] E. Telatar, "Capacity of multi-antenna Gaussian channels," European Transactions on Telecommunications, vol. 10, no. 6, pp. 585-595, 1999.

[28] C. Oestges and B. Clerckx, MIMO Wireless Communications, Elsevier, 2007.

[29] http://sourceforge.net/apps/wordpress/itpp.

[30] W. Hamidouche, R. Vauzelle, C. Olivier, Y. Pousset, and C. Perrine, "Impact of realistic MIMO physical layer on video transmission over mobile ad hoc network," in Proceedings of the IEEE 20th Personal, Indoor and Mobile Radio Communications Symposium (PIMRC' 09), Tokyo, Japan, September 2009. 

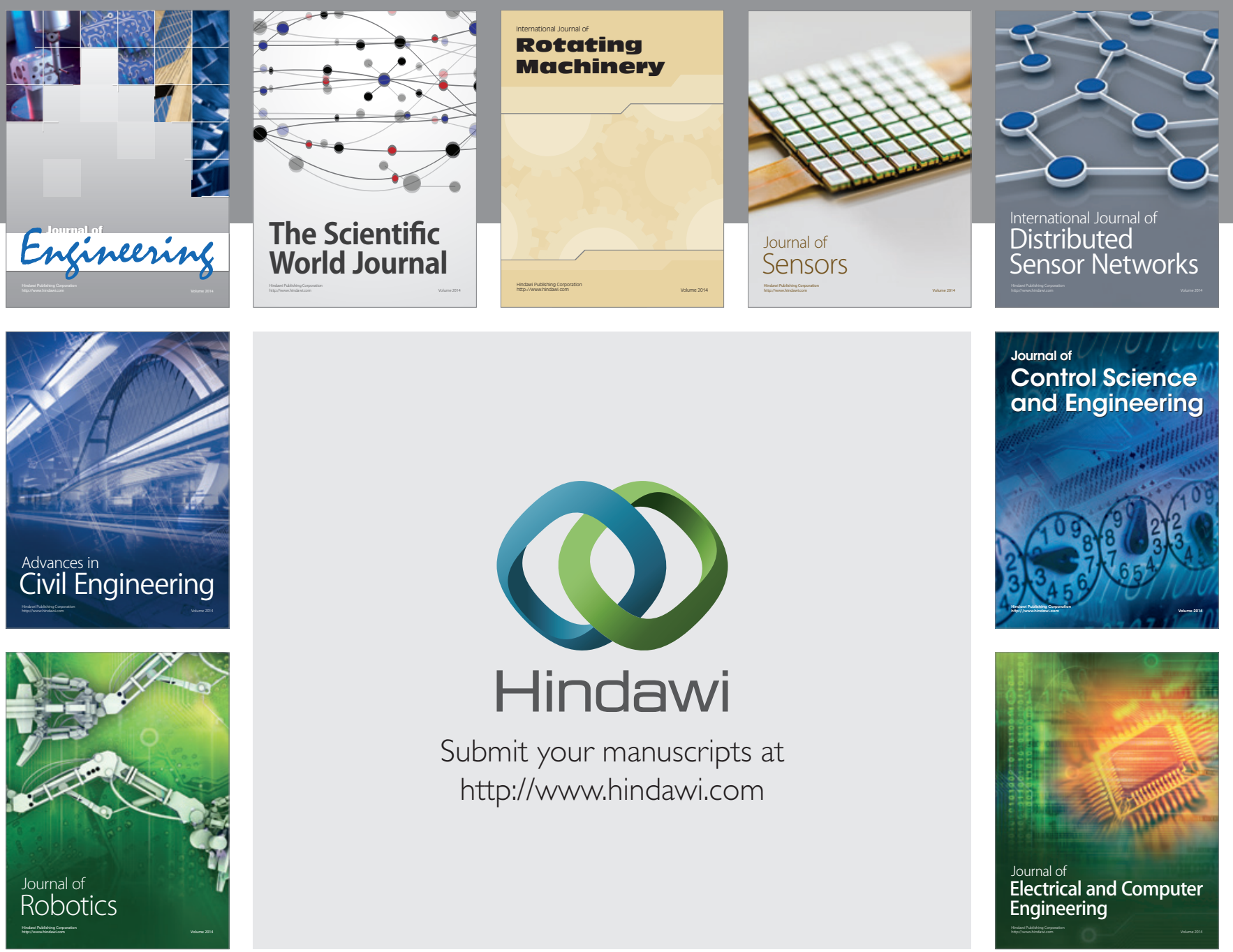

Submit your manuscripts at

http://www.hindawi.com
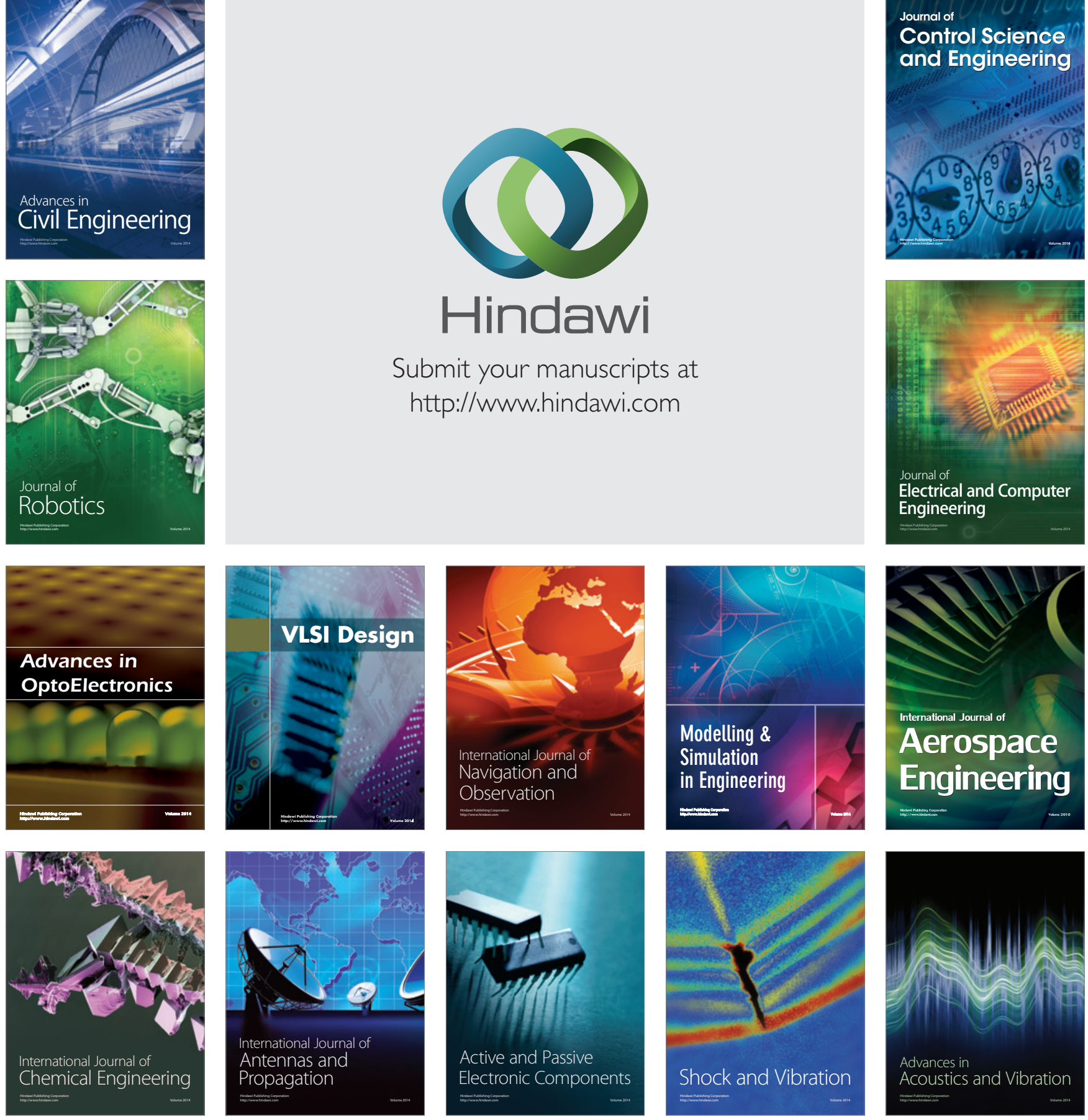